\title{
Poster Abstract: User Authentication using Wrist Mounted Inertial Sensors
}

\author{
Md Abu Sayeed Mondol, Ifat Afrin Emi, Sarah Masud Preum, John A. Stankovic \\ Department of Computer Science, University of Virginia \\ 85 Engineer's Way, P.O. Box 400740 \\ Charlottesville, Virginia 22904-4740 \\ mondol@virginia.edu,iae4qb@virginia.edu,sp9cx@virginia.edu,stankovic@virginia.edu
}

\begin{abstract}
Smart wrist devices available today like smart watches and fitness trackers are usually enriched with inertial sensors such as accelerometers and gyroscopes that can be used to capture wrist motions. This opens an opportunity to use these devices for user authentication, exploiting an important biometric trait of a user: the wrist gestures during performing a signature in the air. In contrast to traditional authentication methods, the gestures can be captured while signing in the air freely with no need for any input media like keypads and signature capture devices. This paper presents result from a preliminary study that shows the potential of the proposed approach to be used for robust user authentication.
\end{abstract}

\section{CCS CONCEPTS}

•Human-centered computing $\rightarrow$ Ubiquitous and mobile computing systems and tools;

\section{KEYWORDS}

Authentication, Smart Watch, Wearable

\section{ACM Reference format:}

Md Abu Sayeed Mondol, Ifat Afrin Emi, Sarah Masud Preum, John A Stankovic. 2016. Poster Abstract: User Authentication using Wrist Mounted Inertial Sensors. In Proceedings of The 16th ACM/IEEE International Conference on Information Processing in Sensor Networks, Pittsburgh, PA USA, April 2017 (IPSN 2017), 2 pages.

DOI: http://dx.doi.org/10.1145/3055031.3055063

\section{INTRODUCTION}

User authentication is a fundamental requirement for allowing access to protected entities or systems in both the physical and the cyber worlds. This includes physical entities like devices, homes and buildings, and cyber systems like logging into applications and services such as email, apps and websites. A strong authentication method is required to allow legitimate users and to prevent attackers from accessing protected information and services. On the other hand, the method needs to be accessible, convenient and easy to use. Smart wrist devices like smart watches and fitness trackers

Permission to make digital or hard copies of all or part of this work for personal or classroom use is granted without fee provided that copies are not made or distributed for profit or commercial advantage and that copies bear this notice and the full citation on the first page. Copyrights for components of this work owned by others than ACM must be honored. Abstracting with credit is permitted. To copy otherwise, or republish, to post on servers or to redistribute to lists, requires prior specific permission and/or a fee. Request permissions from permissions@acm.org.

IPSN 2017, Pittsburgh, PA USA

(C) 2017 ACM. 978-1-4503-4890-4/17/04 . \$\$15.00

DOI: http://dx.doi.org/10.1145/3055031.3055063 are usually enriched with inertial sensors like accelerometers and gyroscopes that are widely used for gesture and activity recognition. The wrist motion of a user while performing a signature is an important biometric trait that can be used to build a strong user authentication system. Though passwords are used widely for user authentication, better protection against attack requires complex passwords that are often inconvenient, particularly for devices like smart phones. Entering a password is also extremely difficult, if not impossible, for devices with very small form factor such as smart watches. The proposed solution can be used conveniently and effectively for user authentication required by different types of entities and systems including smart phones, wearable devices, door locks, appliances, computers, websites, and remote systems. Gesture based user authentication is an active research area. MotionAuth [1] is such a wrist gesture based authentication method that uses only four simple gestures (raising hand, lowering hand, rotation, and circle), and so it is extremely vulnerable to attack. In contrast, signatures are personal, and so signature based methods are more robust against adversary attacks.

The variance among the signatures from a genuine user is the main challenge for any signature based authentication method. Higher variance requires a higher dissimilarity threshold to maintain a reasonable false rejection rate, and vice versa. But a higher dissimilarity threshold makes the system more vulnerable to adversary attacks as the likelihood of a mimicked signature to be accepted by the system increases. This paper presents a method to find the dissimilarity between in-air signatures captured by inertial sensors, and demonstrates results for false rejection rates and false acceptance rates for different dissimilarity thresholds. Results show that nearly $0 \%$ and $2 \%$ false acceptance rates can be achieved for about $35 \%$ and $10 \%$ false rejection rates, respectively.

\section{METHODS}

To provide a signature, a user presses a button on the watch just before and after performing the signature. The noise present in the inertial sensor data is mitigated using an exponential moving average filter. The accelerometers and the gyroscopes used in the wrist device are tri-axial. To verify a signature, the deviation of the signature from a set of template signatures is calculated, and the signature is accepted as genuine only if the deviation is below some predefined threshold. Initially each user provides a set of signatures that are used as templates. In order to address the changes in signature gestures of a user over time, the template set is updated dynamically by replacing a randomly selected signature from the set with an accepted signature of the user. 
Let, $\mu_{i}$ and $\sigma_{i}$ denote the mean and standard deviation of the distances between each pair of signatures from the template set along axis $i$ of the sensors, $\delta_{i}$ be the mean of the distances between an incoming signature and all the template signatures along that axis. Dynamic Time Warping (DTW) is used to measure the distances. Deviation of the incoming signature from the template signatures is defined as:

$$
\text { deviation }=\frac{\sum_{i=1}^{A} \frac{\delta_{i}-\mu_{i}}{\sigma_{i}}}{A}
$$

where $A$ is the total number of axes of the inertial sensors. A signature is accepted only if deviation $\leq T H$, for some threshold TH.

\section{EXPERIMENT}

Accelerometer and gyroscope data for a total of 1334 genuine signatures were collected from 17 participants (10 males and 7 females) using an android powered Sony smart watch. Each participant performed his/her own signature in the air wearing the watch on the dominant hand (all participants in the study are right handed). We also collected 240 adversary signatures where signatures of 4 participants were mimicked by other participants. For each of the participants whose signatures were mimicked, two other participants participated in the mimicking process.

For authentication, there is a trade-off between the acceptance rate of genuine signatures and adversary signatures. The acceptance rates for the signatures depend upon the threshold $(\mathrm{TH})$ value. Figure 1 shows the acceptance rates for both the legitimate signatures and the adversary signatures for different threshold values. As shown on the figure, lower threshold results higher protection against adversary signatures, and vice versa. For example, we can achieve nearly $100 \%$ protection against the adversary attacks by setting the threshold value to 1.5 . However,the acceptance rate of the legitimate signature is only about $65 \%$ for this threshold. On the other hand, we can gain a significant accuracy in the detection of legitimate signatures with a little sacrifice of protection. For instance, if we allow acceptance rate of false signatures to be $1.25 \%$, the acceptance rate of the legitimate signatures increases to about $83 \%$. Even a little more increase in the false acceptance rate to $2.2 \%$ increases the acceptance rate of the legitimate signatures to about $90 \%$. So, the value of the threshold is very critical, and it should be chosen based on the requirement of the target system.

The acceptance rate of the adversary signatures for each of the participants is shown in figure 2 . We provide a unique ID in the range of 1 to 17 to each of the 17 participants. Signatures of participant 1, 5, 7 and 15 were forged for the experiment. According to our observation, the signature pattern of participant 15 was most complex, followed by participant 1, 5 and 7 with participant 7 having the simplest pattern. As shown in the figure, complex signatures are difficult to forge. For example, signature of participant 15 can not be forged even for higher threshold value. Before mimicking a signature, a forger was provided with the written signature of the genuine user. The forger was also allowed to view the gestures both from front and over the shoulder of the genuine user while the latter was signing in the air. Also, the forgers were free to see the signatures and gestures any number of times before or in the

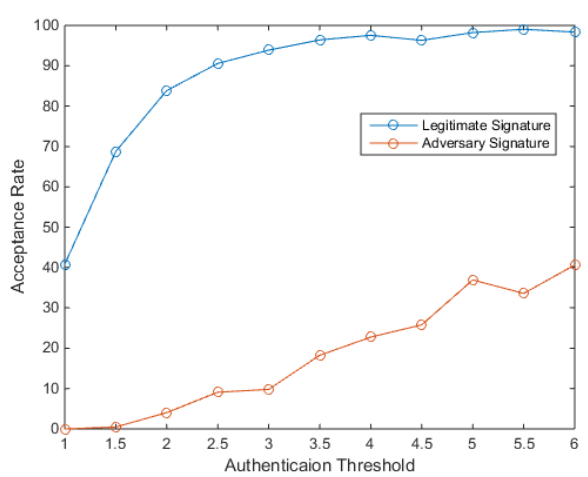

Figure 1: Acceptance rates of the legitimate signatures and the adversary signatures for different values of authentication threshold

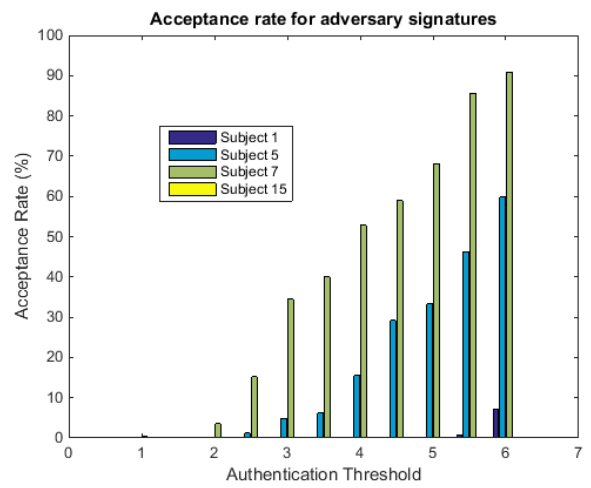

Figure 2: Acceptance rates of adversary signatures

course of forging. For more reliable authentication, a user should use a complex in-air signature that is different from his/her written signature. A pin can be added to the in-air signature to make it more complex. Also, measurements should be taken to prevent potential forgers from viewing the signature gestures.

\section{CONCLUSION}

This paper presents results from a preliminary study on a user authentication method that uses wrist gestures corresponding to in-air signature captured by wrist mounted inertial sensors. Experimental results show that the proposed approach has potential to be used for robust authentication.

\section{ACKNOWLEDGMENTS}

This work was supported, in part, by NSF Grant CNS-1646470 and DGIST Research and Development Program (CPS Global Center) funded by the Ministry of Science, ICT \& Future Planning.

\section{REFERENCES}

[1] Junshuang Yang, Yanyan Li, and Mengjun Xie. 2015. MotionAuth: Motion-based Authentication for Wrist Worn Smart Devices. In Workshop on Sensing Systems and Applications Using Wrist Worn Smart Devices. IEEE. 